\title{
Concentración de hemoglobina glucosilada en pacientes no diabéticas con y sin infección de sitio quirúrgico posterior a la histerectomía
}

\author{
Glycosylated hemoglobin level in patients with and without surgical site infection after \\ hysterectomy
}

\author{
Sebastián Carranza-Lira*, Francisco D. Serrano-Estrada, Eunice López-Muñoz, \\ Luz María Hernández-Jiménez y María Eugenia Chavarría-Olarte \\ Unidad Médica de Alta Especialidad, Hospital de Gineco-Obstetricia "Luis Castelazo Ayala", Instituto Mexicano del Seguro Social, Ciudad de \\ México, México
}

\section{Resumen}

Antecedentes: La infección del sitio quirúrgico (ISQ) se presenta en el 11-12\% de las cirugías. La hemoglobina glucosilada ( $\mathrm{HbA1c}$ ) se ha encontrado significativamente elevada en los pacientes que presentan infección. Objetivo: Comparar la concentración de HbA1c entre pacientes con y sin ISQ posterior a una histerectomía. Método: En mujeres sanas posoperadas de histerectomía total abdominal (abierta) se midió la concentración sérica de HbA1c (normal < 5.7\%) y se comparó la diferencia entre las que presentaron ISQ y las que no. Se investigaron también otros factores de riesgo para ISQ. Se utilizó la prueba $U$ de Mann Whitney. Los valores de HbA1c se estratificaron como normales o anormales, y se contrastaron con la presencia o no de ISQ por medio de la prueba ji al cuadrado. Resultados: Se estudiaron 27 mujeres sin ISQ y 20 con ISQ. La glucosa preoperatoria fue de 88 (70-99) y 86 (70-99) mg/dl para los grupos con y sin ISQ, respectivamente. La HbA1c fue significativamente mayor en el grupo con ISQ (5.6\%; 5-8) que en el grupo sin ISQ (6.5\%; 5.2-8.2). La sensibilidad de la $\mathrm{HbA1C}$ con un punto de corte < 5.7 fue del $80 \%$ y la especificidad fue del 51.9\%. Conclusión: La HbA1c puede servir como criterio pronóstico de ISQ.

Palabras Clave: Infección. Sitio quirúrgico. Hemoglobina glucosilada. Histerectomía.

\begin{abstract}
Background: Surgical site infection (SSI) occurs in 11-12\% of surgeries. The glycosylated hemoglobin (HbA1c) has been found to be significantly elevated in those who presented infection. Objective: To compare the concentration of HbA1c between patients with and without SSI after hysterectomy. Method: In healthy, postoperative women with total abdominal (open) hysterectomy, the HbA1c serum concentration was measured (normal $<5.7 \%$ ) and the difference between those who presented SSI and other risk factors for SSI were compared with Mann Whitney $U$ test was used. The HbA1C values were stratified as normal or abnormal and were contrasted with the presence or absence of SSI by means of X2. Results: 27 women without SSI and 20 with SSI were studied. The preoperative glucose was and 88 (70-99) mg/dl and 86 (70-99) mg/dl for the groups
\end{abstract}

\section{Correspondencia:}

*Sebastián Carranza-Lira

Puente de Piedra, 150-422, Torre 1

Col. Toriello Guerra

Fecha de recepción: 03-07-2019

C.P. 14050, Tlalpan, Ciudad de México, México

E-mail: drsebastiancarranza@gmail.com
Cir Cir. 2020;88(3):344-348

Contents available at PubMed www.cirugiaycirujanos.com (http://creativecommons.org/licenses/by-nc-nd/4.0/). 
with and without SSI respectively. The HbA1C was significantly higher in the group with ISQ 5.6\% (5-8) vs. 6.5\% (5.2-8.2). The sensitivity of $\mathrm{HbA} 1 \mathrm{C}$ with cut point $<5.7$ was $80 \%$ and the specificity was $51.9 \%$. Conclusion: $\mathrm{HbA1c}$ can serve as a prognostic criterion of ISQ.

Key Words: Infection. Surgical site. Glycosylated hemoglobin. Hysterectomy.

\section{Introducción}

La infección del sitio quirúrgico (ISQ) se presenta en el $11-12 \%$ de las cirugías realizadas, mientras que para las histerectomías esta frecuencia es del $1.7 \% \%^{1,2} ; \sin$ embargo, puede estar subestimada dado que muchas veces la infección se manifiesta una vez egresada la paciente ${ }^{3}$.

La ISQ puede ser superficial, profunda, de órgano o en los espacios intraperitoneal o retroperitoneal. La ISQ superficial es aquella que ocurre en los primeros 30 días de la cirugía y está limitada a la piel y al tejido subcutáneo, más alguno de los siguientes criterios: descarga purulenta, cultivo positivo de la secreción del sitio quirúrgico o cuando el clínico abre una incisión que es dolorosa, blanda, hinchada, eritematosa o caliente. La infección profunda es aquella que ocurre en los 30 días posteriores a la cirugía y que involucra tejidos blandos profundos, tales como la fascia y el músculo. Aquellas que se presentan en órganos o en espacios lo hacen también en los 30 días posteriores a la cirugía, excluyendo los planos superficiales y profundos ${ }^{2,4,5}$. En dos terceras partes de los casos las infecciones son superficiales, y en ginecología la más frecuente es la celulitis en el sitio de incisión y en la cúpula vaginal ${ }^{6}$. Es conveniente mencionar que la histerectomía se considera una cirugía limpia contaminada. En la Unidad Médica de Alta Especialidad Hospital de Gineco-Obstetricia Luis Castelazo Ayala, en el año 2017, según los registros del servicio de ginecología, se realizaron 1,067 histerectomías totales abdominales, de las cuales 72 tuvieron infección superficial de la herida, 9 profunda y 20 de órgano y espacios.

Existen múltiples factores que intervienen en la génesis de la infección, tales como la edad, la obesidad", la diabetes, el tabaquismo, las infecciones en otro sitio del organismo, la colonización por otros tipos de microorganismos, la respuesta inmunitaria alterada, la duración de la cirugía, la duración del cepillado quirúrgico, la antisepsia de la piel de la pared abdominal (clorhexidina vs. yodopovidona) ${ }^{8}$, el aseo vaginal prequirúrgico ${ }^{9}$, el rasurado o no rasurado preoperatorio, la profilaxis antimicrobiana ${ }^{7}$, la ventilación en el quirófano, la esterilización del instrumental, los cuerpos extraños en herida quirúrgica, los drenajes, la hemostasis, la falla en obliterar el espacio muerto (cierre o no del tejido celular subcutáneo) ${ }^{10}$, el grosor del tejido celular subcutáneo $>3 \mathrm{~cm}^{11}$ y el trauma tisular ${ }^{12}$.

Las infecciones son más comunes en el paciente diabético ${ }^{13}$ (el $21 \%$ de los pacientes con sepsis son diabéticos $\left.{ }^{14}\right)$. La hiperglucemia en las primeras 48 horas de la cirugía se ha asociado a mayor riesgo de infección ${ }^{15}$; se ha reportado que una glucosa $>12$ $\mathrm{mmol} / \mathrm{l}$ en el primer día de posoperatorio incrementa seis veces el riesgo de infección nosocomial ${ }^{16}$. Los mecanismos para explicar este incremento en el riesgo de infección son, entre otros, la disminución de la quimiotaxis de polimorfonucleares ${ }^{17}$, la menor producción de superóxidos con relación al incremento de las concentraciones de glucosa ${ }^{18}$, las alteraciones en la fagocitosis ${ }^{19}$, los defectos en la opsonización $n^{13,20}$, los defectos en la quimiotaxis de los monocitos ${ }^{21}$ y el incremento de la expresión de CD152 por los linfocitos $T$, que se sabe que regula a la baja la respuesta inmunitaria ${ }^{22}$.

La hemoglobina glucosilada ( $\mathrm{HbA1c})$ es un indicador del control metabólico de los últimos 3 meses ${ }^{23,24}$. En estudios recientes se ha analizado la utilidad de medir la $\mathrm{HbA} 1 \mathrm{c}$ antes de la cirugía con la finalidad de detectar pacientes diabéticos no tratados o con pobre control, y se ha encontrado que está significativamente elevada en los que presentaron infección ${ }^{25}$. Asimismo, en la cirugía de pie y tobillo la HbA1c estuvo relacionada directamente con el riesgo de infección, que era un $15 \%$ más alto en este grupo ${ }^{26}$. Sin embargo, en el hospital no existe un registro de cuantas pacientes histerectomizadas con ISQ tienen alterada la HgbA1c, ya que esta que no forma parte de los estudios preoperatorios sistemáticos.

Por todo lo anterior, el objetivo de este estudio fue comparar la concentración de HbA1c entre pacientes con y $\sin I S Q$ tras una histerectomía.

\section{Método}

Estudio prospectivo, transversal y comparativo realizado en mujeres no diabéticas sin infección 
cervicovaginal posoperadas de histerectomía total abdominal (abierta). Previa firma del consentimiento informado, a todas las pacientes se les tomó una muestra de $5 \mathrm{ml}$ de sangre periférica en un tubo con EDTA para medir la concentración de HbA1c, lo cual se realizó por inhibición de la inmunoaglutinación en látex utilizando estuches comerciales (Hemoglobin A1c, Reagent Kit, Ref. 10698915. Siemens DCA Systems). El valor de la HbA1c se consideró normal cuando fue $<5.7 \%$ y anormal cuando fue $\geq 5.7 \%$. Además, se investigaron los siguientes datos: edad, comorbilidad (como hipertensión arterial sistémica), tabaquismo, consumo excesivo de alcohol, cirugías previas, índice de masa corporal (IMC, peso en $\mathrm{kg} /$ talla en $\mathrm{m}^{2}$ ), grosor del panículo adiposo, sangrado transoperatorio y tiempo quirúrgico. Todas las pacientes recibieron profilaxis antibiótica con cefuroxima (750 mg en dosis única), así como una dosis de metronidazol vaginal la noche previa a la cirugía; el antiséptico utilizado para el aseo prequirúrgico de la pared abdominal y de la vagina fue yodopovidona. Posteriormente a la cirugía se vigiló la evolución clínica de la paciente y se consignó la presencia o no de ISQ durante su internamiento y en los 30 días posteriores a la intervención. En aquellas con ISQ, se especificó el tipo de infección.

Se analizaron los resultados preoperatorios de hemoglobina $(\mathrm{g} / \mathrm{dl})$, leucocitos $\left(10^{3} / \mu \mathrm{l}\right)$, plaquetas $\left(10^{3} / \mu \mathrm{l}\right)$, neutrófilos $\left(10^{3} / \mu \mathrm{l}\right)$, linfocitos $\left(10^{3} / \mu \mathrm{l}\right)$, monocitos $\left(10^{3} / \mu \mathrm{l}\right)$, eosinófilos $\left(10^{3} / \mu \mathrm{l}\right)$, basófilos $\left(10^{3} / \mu \mathrm{l}\right)$ y concentraciones de glucosa; estas últimas tenían que ser $\leq 100 \mathrm{mg} / \mathrm{dl}$ para que las pacientes fueran incluidas en el estudio.

El tamaño de la muestra se calculó considerando un error tipo alfa de 0.05 y una potencia del $80 \%$, con una diferencia entre las medias de 0.83 y una desviación estándar para el grupo I de 0.95 y para el grupo II de 0.76 , quedando 23 pacientes en el grupo I y 17 pacientes en el grupo II (Medcalc V 18.5).

La comparación entre los grupos se realizó con la prueba U de Mann Whitney. Posteriormente, los valores de $\mathrm{HbA1c}$ se estratificaron como normales $\mathrm{o}$ anormales, y se contrastaron con la presencia o no de ISQ por medio de la prueba de ji al cuadrado. La sensibilidad y la especificidad se calcularon utilizando las curvas ROC. Las variables que resultaron significativamente asociadas con el estado de infección se introdujeron en un modelo de regresión logística.

El protocolo fue aceptado por el Comité Local de Investigación en Salud y por el Comité de Ética en Investigación con el número R-2018-3606-017, y las pacientes firmaron la hoja de consentimiento informado.
Tabla 1. Datos generales de las mujeres sin y con infección del sitio quirúrgico

\begin{tabular}{lccc}
\hline & $\begin{array}{c}\text { Sin infección del } \\
\text { sitio quirúrgico }\end{array}$ & $\begin{array}{c}\text { Con infección del } \\
\text { sitio quirúrgico }\end{array}$ & $\mathbf{p}$ \\
\hline Edad (años) & $46(31-54)$ & $47(40-74)$ & 0.141 \\
Peso $(\mathrm{kg})$ & $73.0(58-84)$ & $72.0(58-82)$ & 0.597 \\
Talla $(\mathrm{m})$ & $1.53(1.43-1.64)$ & $1.52(1.43-1.64)$ & 0.762 \\
Índice de masa & $29.5(24.0-38.3)$ & $30.9(21.6-34.7)$ & 0.974 \\
corporal & & & \\
\hline
\end{tabular}

Tabla 2. Resultados de la biometría hemática y de la glucosa en mujeres sin y con infección del sitio quirúrgico

\begin{tabular}{lccc}
\hline & $\begin{array}{c}\text { Sin infección del } \\
\text { sitio quirúrgico }\end{array}$ & $\begin{array}{c}\text { Con infección del } \\
\text { sitio quirúrgico }\end{array}$ & $\mathbf{p}$ \\
\hline Hemoglobina $(\mathrm{g} / \mathrm{dl})$ & $13.5(11-16)$ & $13.5(11-16)$ & 0.613 \\
Leucocitos $\left(10^{3} / \mu \mathrm{l}\right)$ & $7.8(5.4-13.0)$ & $8.5(5.4-13.0)$ & 0.483 \\
Plaquetas $\left(10^{3} / \mu \mathrm{l}\right)$ & $250.0(150-507)$ & $214.0(162-507)$ & 0.262 \\
Neutrófilos $\left(10^{3} / \mu \mathrm{l}\right)$ & $60.0(50.0-87.0)$ & $64.0(50.0-87.0)$ & 0.309 \\
Linfocitos $\left(10^{3} / \mu \mathrm{l}\right)$ & $16.0(6.0-70.0)$ & $16.0(6.0-70.0)$ & 0.713 \\
Monocitos $\left(10^{3} / \mu \mathrm{l}\right)$ & $7.0(4.0-19.0)$ & $6.5(4.0-19.0)$ & 0.974 \\
Eosinófilos $\left(10^{3} / \mu \mathrm{l}\right)$ & $1.0(0.1-5.0)$ & $1.0(0.1-5.0)$ & 0.303 \\
Basófilos $\left(10^{3} / \mu \mathrm{l}\right)$ & $0.900(0.3-5.0)$ & $0.800(0.3-5.0)$ & 0.559 \\
Glucosa $(\mathrm{mg} / \mathrm{dl})$ & $86.0(70-99)$ & $90.0(70-99)$ & 0.093 \\
\hline
\end{tabular}

\section{Resultados}

Se estudiaron 27 mujeres sin ISQ y 20 con ISQ; de estas últimas, en 18 hubo infección de piel y de tejido celular subcutáneo, y en las otras dos abarcó además la aponeurosis. No se encontró diferencia entre los grupos en cuanto a edad, peso, talla e IMC (Tabla 1). Con respecto a los antecedentes de hipertensión, tabaquismo y consumo de alcohol, no hubo diferencias entre los grupos. La mujeres con ISQ tuvieron con más frecuencia el antecedente de cirugía previa (75 vs. $25 \% ; p<0.016$ ). El sangrado transoperatorio fue en ambos grupos de $250 \mathrm{ml}$ (100-400). El grosor del panículo adiposo, el sangrado transoperatorio y el tiempo quirúrgico no mostraron diferencias entre los grupos. La hemoglobina, los leucocitos, la cuenta diferencial y las plaquetas no fueron diferentes entre los grupos (Tabla 2). La concentración de glucosa preoperatoria fue de 88 (70-99) mg/dl en el grupo con ISQ y de 86 (70-99) mg/dl en el grupo sin ISQ, sin diferencia significativa entre ellos. La HbA1c fue significativamente mayor en el grupo con ISQ, 6.5\% (5.2-8.2), en comparación con el 5.5\% (5-8) en el 
grupo sin ISQ ( $p<0.002)$. La HbA1c estuvo un 18\% más elevada en el grupo con ISQ.

La proporción de mujeres con IMC > 27 y HbA1c elevada fue mayor en el grupo con ISQ (63.2 vs. $55.63 \%$; $p=0.476$ ), pero sin significancia estadística. La proporción de mujeres con ISQ fue mayor en aquellas con HbA1c elevada que en aquellas con HbA1c normal ( 80 vs. $20 \% ; p<0.036$ ). No hubo diferencia en la proporción de mujeres sin y con ISQ e IMC > 27 (81.5 vs. $80 \% ; p=0.593)$.

La sensibilidad de la HbA1c con punto de corte en $<5.7$ fue del $80 \%$ y la especificidad fue del $51.8 \%$; el valor predictivo positivo (VPP) fue del $55.2 \%$ y el valor predictivo negativo (VPN) fue del $77.8 \%$. Con un punto de corte $<6.15$, la sensibilidad fue del $70 \%$ y la especificidad del $77.8 \%$; el VPP fue del $70 \%$ y el VPN fue del $77.8 \%$. Considerando un punto de corte $<6.15$, la sensibilidad fue del $70 \%$ y la especificidad del $78 \%$; el VPP fue del $70 \%$ y el VPN fue del $77.8 \%$. En el análisis de regresión logística conservaron significancia estadística el antecedente de cirugía (7.7; intervalo de confianza del 95\% [IC 95\%]: 1.50-39.3; $p<0.014$ ) y la HbA1c $\geq 5.7$ (5.1; IC 95\%: 1.1-22.9; $p<0.033)$ como predictores de infección.

\section{Discusión}

En estudio se evaluaron las concentraciones de $\mathrm{HbA1c}$ en mujeres histerectomizadas con y sin ISQ. Esta evaluación fue hecha en el posoperatorio asumiendo que el valor obtenido era un reflejo del estado metabólico.

En la génesis de la ISQ intervienen distintos factores, entre ellos el control metabólico ${ }^{15,16}$. En las mujeres sin diabetes, la elevación de la $\mathrm{HbA} 1 \mathrm{c}$ puede revelar alteraciones discretas de la concentración de glucosa, las cuales son desconocidas por las pacientes, que no se saben con alteración en el metabolismo de los carbohidratos, pero que sin embargo incrementan el riesgo de infección al modificar la respuesta inmunitaria y la funcionalidad de los leucocitos ${ }^{17-22}$.

En este estudio, al analizar factores como edad, el IMC, el grosor del panículo adiposo, el tiempo quirúrgico, el sangrado transquirúrgico, el uso de profilaxis antibiótica, la antisepsia prequirúrgica y la ausencia de infección cervicovaginal no se encontró diferencia entre los grupos, lo que permitió considerar a la HbA1c como un auxiliar que debería considerarse al valorar el riesgo de ISQ.

En este estudio, la $\mathrm{HbA} 1 \mathrm{c}$ estuvo un $18 \%$ más elevada en el grupo con ISQ, de modo similar a lo reportado en otro estudio en el que los pacientes con infección la tuvieron un $15 \%$ más elevada ${ }^{26}$.

La sensibilidad de la $\mathrm{HbA} 1 \mathrm{c}$ con un punto de corte en $<5.7$ fue del $80 \%$ y la especificidad fue del $51.8 \%$, con un VPP del $55.2 \%$ y un VPN del $77.8 \%$, lo cual es adecuado, ya que al desear predecir el riesgo de ISQ la sensibilidad es el parámetro más apropiado.

Este estudio tuvo como fortaleza que ambos grupos eran homogéneos, salvo por el antecedente de cirugía, pero permitió darle el peso específico a la HbA1c. Como debilidad, el tamaño de la muestra es pequeño, por lo que deberá reclutarse un número mayor de pacientes y validar estos resultados antes de incluir la determinación de la HbA1c como parte de la evaluación preoperatoria.

Todo lo anterior permite concluir que la HgA1c puede ser de utilidad para predecir el riesgo de ISQ en las pacientes en que se realizará una histerectomía total abdominal.

\section{Conflicto de intereses}

Los autores declaran que no existe conflicto de intereses.

\section{Responsabilidades éticas}

Protección de personas y animales. Los autores declaran que para esta investigación no se han realizado experimentos en seres humanos ni en animales.

Confidencialidad de los datos. Los autores declaran que han seguido los protocolos de su centro de trabajo sobre la publicación de datos de pacientes.

Derecho a la privacidad y consentimiento informado. Los autores han obtenido el consentimiento informado de los pacientes y/o sujetos referidos en el artículo. Este documento obra en poder del autor de correspondencia.

\section{Bibliografía}

1. Kamat $A A$, Brancazio $L$, Gibson M. Wound infection in gynecologic surgery. Infect Dis Obstet Gynecol. 2000;8:230-4.

2. Black JD, de Haydu C, Fan L, Sheth SS. Surgical site infections in gynecology. Obstet Gynecol Surv. 2014;69:501-10.

3. Lake AG, McPencow AM, Dick-Biascoechea MA, Martin DK, Erekson EA. Surgical site infection after hysterectomy. Am J Obstet Gynecol. 2013;209:490.e1-9.

4. Horan TC, Gaynes RP, Martone WJ, Jarvis WR, Emori TG. CDC definitions of nosocomial surgical site infections, 1992: a modification of CDC definitions of surgical wound infections. Infect Control Hosp Epidemiol. 1992;13:606-8.

5. Mangram AJ, Horan TC, Pearson ML, Silver LC, Jarvis WR. Guideline for prevention of surgical site infection, 1999. Centers for Disease Control and Prevention (CDC) Hospital Infection Control Practices Advisory Committee. Am J Infect Control. 1999;27:97-132.

6. Lazenby GB, Soper DE. Prevention, diagnosis, and treatment of gynecologic surgical site infections. Obstet Gynecol Clin North Am. 2010;37:379-86. 
7. Molina-Cabrillana J, Valle-Morales L, Hernández Vera, López-Carrió I, García-Hernández JA, Bolaños-Rivero M. Surveillance and risk factors on hysterectomy wound infection rate in Gran Canaria, Spain. Eur J Obstet Gynecol Reprod Biol. 2008;136:232-8

8. Dumville JC, McFarlane E, Edwards P, Lipp A, Holmes A, Liu Z. Preoperative skin antiseptics for preventing surgical wound infections after clean surgery. Cochrane Database Syst Rev 2015;21:CD003949.

9. Culligan PJ, Kubik K, Murphy M, Blackwell L, Snyder J. A randomized trial that compared povidone iodine and chlorhexidine as antiseptics for vaginal hysterectomy. Am J Obstet Gynecol. 2005;192:422-5.

10. Gurusamy KS, Toon CD, Davidson BR. Subcutaneous closure versus no subcutaneous closure after non-cesarean surgical procedures. Cochrane Database Syst Rev 2014;1:CD010425.

11. Soper DE, Bump RC, Hurt WC. Wound infection after abdominal hysterectomy: effect of the depth of subcutaneous tissue. Am J Obstet Gynecol. 1995;173:465-71.

12. Jaiyeoba O. Postoperative infections in obstetrics and gynecology. Clin Obstet Gynecol. 2012;55:904-13.

13. Peleg AY, Weerarathna T, McCarthy JS, Davis TM. Common infections in diabetes: pathogenesis, management and relationship to glycaemic control. Diabetes Metab Res Rev. 2007;23:3-13.

14. Koh GC, Peacock SJ, van der Poll T, Wiersinga WJ. The impact of diabetes on the pathogenesis of sepsis. Eur J Clin Microbiol Infect Dis 2012;31:379-88.

15. Latham R, Lancaster AD, Covington JF, Pirolo JS, Thomas CS Jr. The association of diabetes and glucose control with surgical-site infections among cardiothoracic surgery patients. Infect Control Hosp Epidemiol. 2001;22:607-12.

16. Pomposelli J J, Baxter JK $3^{\text {rd }}$, Babineau TJ, et al. Early postoperative glucose control predicts nosocomial infection rate in diabetic patients. J Parenter Enteral Nutr. 1998;22:77-81.
17. Delamaire M, Maugendre D, Moreno M, Le Goff MC, Allannic $H$, Genetet B. Impaired leucocyte functions in diabetic patients. Diabet Med. 1997;14:29-34.

18. Mazade MA, Edwards MS. Impairment of type III group B Streptococcus-stimulated superoxide production and opsonophagocytosis by neutrophils in diabetes. Mol Genet Metab. 2001;73:259-67.

19. Alexiewicz JM, Kumar D, Smogorzewski M, Klin M, Massry SG. Polymorphonuclear leukocytes in non-insulin-dependent diabetes mellitus: abnormalities in metabolism and function. Ann Intern Med. 1995; 123:919-24.

20. Hostetter MK. Handicaps to host defense. Effects of hyperglycemia on C3 and Candida albicans. Diabetes. 1990;39:271-5.

21. Hill HR, Augustine NH, Rallison ML, Santos Jl. Defective monocyte chemotactic responses in diabetes mellitus. J Clin Immunol. 1983;3:70-7.

22. Spatz M, Eibl N, Hink S, et al. Impaired primary immune response in type-1 diabetes. Functional impairment at the level of APCs and T-cells. Cell Immunol. 2003;221:15-26.

23. MedlinePlus. Prueba de hemoglobina glicosilada ( $\mathrm{HbA1c})$. (Consultado el 31 de mayo de 2018.) Disponible en: https://medlineplus.gov/spanish/ a1c.html

24. Goldstein DE, Little RR, Wiedmeyer HM, England JD, McKenzie EM. Glycated hemoglobin: methodologies and clinical applications. Clin Chem. 1986;32(10 Suppl):B64-70.

25. Gatti G, Perrotti A, Reichart D, Maschietto L, Onorati F, Chocron S. Glycated hemoglobin and risk of sternal wound infection after isolated coronary surgery. Circ J. 2017;81:36-43.

26. Humphers JM, Shibuya N, Fluhman BL, Jupiter D. The impact of glycosylated hemoglobin and diabetes mellitus on wound-healing complications and infection after foot and ankle surgery. J Am Podiatr Med Assoc. $2014 ; 104: 320-9$ 